\title{
Masked transposition effects for simple versus complex nonalphanumeric objects
}

\author{
Javier García-Orza • Manuel Perea • \\ Alejandro Estudillo
}

Published online: 8 September 2011

(C) Psychonomic Society, Inc. 2011

\begin{abstract}
When two letters/digits/symbols are switched in a string (e.g., jugde-judge; 1492-1942; *?\&\&-*\$?), the resulting strings are perceptually similar to each other and produce a sizable masked transposition priming effect with the masked priming same-different matching task. However, a parallel effect does not occur for strings of pseudoletters (e.g., $\mathbb{\square} \llbracket \pm-\mathbb{-} \llbracket \llbracket \llbracket 1$; García-Orza, Perea, \& Muñoz, Quarterly Journal of Experimental Psychology, 63, 1603$1618,2010)$. In the present study, we examined whether masked transposition priming is specific to alphanumeric stimuli or whether it also occurs with strings composed of other "objects" - namely, line drawings of common objects (Experiment 1) and geometrical shapes (Experiment 2). Results showed a significant masked transposition priming effect for geometrical shapes (e.g., $\mathbf{\square} \star \mathbf{\Delta} \mathbf{D}-\mathbf{\square} \mathbf{\Delta} \star \mathbf{D}$ ), but not for line drawings of common objects (e.g., ar $\theta$ - These findings suggest that the mechanism involved in the coding of position in masked priming works only with perceptually simple, familiar "objects" (i.e., letters, numbers, symbols, or geometrical shapes), once their identities have been well ascertained.
\end{abstract}

\section{Keywords Priming - Visual word recognition · Attention}

One topic that has attracted a great amount of attention in past years is how the cognitive system encodes letter

J. García-Orza $(\triangle) \cdot$ A. Estudillo

Departamento de Psicología Básica, Facultad de Psicología,

Universidad de Málaga,

Campus de Teatinos, $\mathrm{s} / \mathrm{n}$,

29071 Málaga, Spain

e-mail: jgorza@uma.es

M. Perea

Universitat de València,

Valencia, Spain position (see, e.g., Davis, 2010; Whitney, 2008). When reading words, we need to identify not only the constituent elements (i.e., the letters), but also their positions within the string; if not, causal and casual would not be distinguished. Most of the experiments on this issue have employed the masked priming paradigm (Forster \& Davis, 1984), which is a highly useful technique for unveiling the earliest stages of processing (see Grainger, 2008, for a review). The central finding is that a target word is recognized faster when it is preceded by a briefly presented (around $50 \mathrm{~ms}$ ) masked transposed-letter nonword prime (e.g., jugde$J U D G E$ ) than when it is preceded by a orthographic control (jupte-JUDGE) (Perea \& Lupker, 2003b, 2004; for further evidence, see also Guerrera \& Forster, 2007; Schoonbaert \& Grainger, 2004). That is, the transposed-letter nonword $J U G D E$ may initially activate the lexical representation of $J U D G E$; indeed, in a masked priming paradigm, jugdeCOURT is responded to faster than ocaen-COURT (see Perea \& Lupker, 2003a).

One critical question here is whether these masked transposition priming effects reflect a position-coding mechanism that is specific to letters or whether they reflect a more general mechanism. Recent research has shown that masked transposition priming can be obtained with strings of digits (e.g., 3745-3475 faster than 3825-3475; GarcíaOrza \& Perea, 2011; García-Orza, Perea, \& Muñoz, 2010) and with strings of well-known symbols (e.g., *? \$\&_*\$? faster than $* \% \pm \&-* \$ ? \&$; García-Orza et al., 2010). Thus, at the early moments of processing, the cognitive system employs a rather flexible position coding not only for strings of letters, but also for strings of digits and symbols.

How does the brain initially encode positions for letters, digits, or symbols in a string? One common assumption in the literature on memory and visual attention is the presence of position uncertainty among series of objects 
in the initial encoding of a visual scene (see, e.g., Ashby, Prinzmetal, lvry, \& Maddox, 1996; Logan, 1996; Ratcliff, 1981): Ratcliff indicated that strings of letters "have distributions over position so that the representation of one letter will extend into adjacent letter positions" (p. 554); at a more general level, Logan indicated that "the representation of location is distributed across space"; and Ashby et al. assumed that there is location "uncertainty about the location of visual features" (p. 166). The assumption of position uncertainty is central in Gomez, Ratcliff, and Perea's (2008) overlap model of letter position coding (for a similar view, see also Davis, 2010; Norris, Kinoshita, \& van Casteren, 2010). In the overlap model, location of objects (e.g., digits, letters) within a string is understood as distributions along a dimension. That is, upon presentation of JUGDE (i.e., a string of letters), the letter (object) representations of $J, U, G$, $D$, and $E$ would be activated, and more importantly, there would be some degree of perceptual uncertainty regarding the position of each letter (object), so that $G$ in the third position would partially activate the neighboring letter positions; this is why JUGDE may be perceived initially as $J U D G E$. Importantly, in the overlap model, perceptual uncertainty would occur at a general-domain level, rather than at the letter level, so that masked transposition priming at the earliest stages of processing could be similar for strings of letters, digits, symbols, and other objects. Obviously, we acknowledge that the processing of letters and the processing of nonlinguistic objects differ at some processing stage (e.g., Farah, 1994; Humphreys \& Rumiati, 1998; Schendan, Ganis, \& Kutas, 1998; Tydgat \& Grainger, 2009), but the point here is that, in the overlap model, a general-domain mechanism would be used to initially encode the position of letters and other objects within a string. Alternatively, other models of letter position coding explicitly assume that mechanisms responsible for the effects of letter transposition are domain specific (LCD model, Dehaene, Cohen, Sigman, \& Vinckier, 2005; SERIOL model, Whitney, 2001, and Whitney \& Cornelissen, 2005). Specifically, the SERIOL and LCD models assume that the similarity of two letter strings is based mainly on the number of open bigrams (ordered pairs of letters; e.g., $j u, j d$, etc.) shared by the two stimuli (Whitney, 2001; see also Dehaene et al., 2005). In these two models, the visual word form area would be responsible for the activation of bigrams; in other words, this language-specific area would be mainly responsible for transposed-letter effects. Given that this area is considered to be specific to letter processing (Baker et al., 2007; see also Cohen et al., 2000), the nature of masked transposition priming would be qualitatively different for letters and for other "objects."

Importantly, masked transposition priming does not occur for all strings of objects. García-Orza et al. (2010) failed to find any signs of a masked transposition effect for

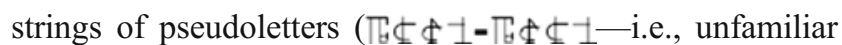
objects). Likewise, Perea, Abu Mallouh, García-Orza, and Carreiras (2011) failed to obtain masked transposition priming for strings of Arabic letters when the individuals had no knowledge of Arabic letters (i.e., these letters acted as "pseudoletters" for the participants). Importantly, intermediate learners of Arabic (i.e., individuals who were already familiar with Arabic letters) did show a masked transposition priming effect (Perea et al., 2011). Taken together, these findings suggest that "object" identity must be well ascertained for masked transposition priming to occur.

The goals of the present study are (1) to examine whether the cognitive system encodes "object" position of nonalphanumeric objects within a string at the earliest moments of processing, using the same masked priming paradigm as Garcia-Orza et al. (2010) and Perea et al. (2011), and (2) to examine whether the perceptual complexity of the constituent objects modulates this phenomenon. This will allow us to observe the similarities and differences of the position-encoding processes for alphanumeric versus nonalphanumeric objects (note that the presence of a similar pattern of data for alphanumeric and nonalphanumeric would go against models that defend the existence of a specific mechanism devoted to encoding alphanumeric stimuli). To examine the earliest stages of "object" (letter, digit, etc.) position coding, a highly useful task is the masked priming same-different judgment task (for reviews of this task, see Kinoshita \& Norris, 2009; Perea, Moret-Tatay, \& Carreiras, in press). Unlike lexical decision or naming, this task can be applied to a vast variety of stimuli-not just alphanumeric stimuli. In a same-different matching task, a probe (e.g., house) and a target (e.g., HOUSE) are presented sequentially. The participant's task is to decide whether the probe and target are the same stimulus or not. Prior research has shown that, for same trials, responses are faster when the target stimulus is preceded by a masked transposed-letter prime (e.g., hosueHOUSE) than when the prime is a replacement-letter control (e.g., horae-HOUSE) (Norris \& Kinoshita, 2008; see also Perea \& Acha, 2009). Using this task, García-Orza et al. (2010; see also García-Orza \& Perea, 2011) found masked transposition priming effects, on same responses, of similar magnitude with pseudowords (e.g., ERTI), strings of consonants (e.g., FGHT), strings of digits (e.g., 6745), and strings of symbols (e.g., *?\$\&). (As usual, masked priming effects did not occur for different responses, since both related primes and control primes provide similar evidence supporting the hypothesis that the target is different from the probe [e.g., probe-prime-target; compare $L M N T-B T P V-$ $B T P V$ vs. LMNT-JGRW-BTPV; see Norris \& Kinoshita, 2008; Perea et al., in press].) In contrast, García-Orza et al. (2010) found no signs of a masked transposition priming effect with strings of pseudoletters (e.g., $₫ \mathbb{T} \llbracket \pm$ ) (i.e., 
unfamiliar objects; see also Perea et al., 2011, for a similar finding with Arabic letters for readers unfamiliar with this script).

To sum up, masked transposition priming occurs not only for strings of letters, but also for strings of other familiar "objects" (i.e., numbers, symbols). However, masked transposition priming does not occur when the "objects" are unfamiliar (i.e., strings of pseudoletters and strings of letters from an unknown script). The present experiments were designed to examine the generality of the position-coding mechanism at the earliest stages of processing by employing strings of nonalphanumeric objects (instead of alphanumeric objects) in a masked priming paradigm. More specifically, we focused on two issues: (1) whether masked transposition priming could be found with strings of familiar nonalphanumeric objects and (2) whether the magnitude of masked transposition priming would be modulated by the complexity of the constituent objects. We examined these two issues by employing strings of two types of familiar nonalphanumeric objects that differed in their complexity: line drawings of common objects (e.g., $\%$ \& $\square$ ) and geometrical shapes (e.g., $\Delta \mathbf{D} \star$ ). In Experiment 1, masked transposition priming was tested using line drawings of common objects (e.g.,

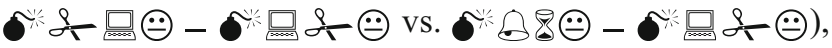
whereas in Experiment 2, we employed simple geometrical shapes (e.g., $\Delta D \star-\Delta D \star$ vs. $\Delta O \square \star-\Delta D \star)$ ). As in the García-Orza et al. (2010) experiments, the prime stimuli were generated by transposing or replacing two adjacent "objects" in the target stimulus (initial: e.g.,

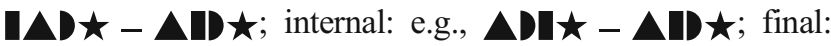
$\Delta \star \mathbf{D}-\boldsymbol{\Delta} \boldsymbol{\Delta} \star \mathbf{D}$ ). As additional controls, we also included unrelated and identity primes in the experiments. The reason is that the replacement condition may conflate the effect of change in object position with a change in object identity. To examine the effect of object position independently of object identity, the identity priming condition is a useful baseline for the transposition primes (see Kinoshita, Castles, \& Davis, 2009); furthermore, the unrelated condition can also be employed as a baseline for the substitution primes (Perea \& Lupker, 2004; see also García-Orza et al. 2010; Kinoshita et al., 2009; Perea \& Acha, 2009). To avoid physical continuity between primes and targets, primes were presented in smaller size than the targets. This guaranteed complete visual masking of the primes by the targets and also made the primes and the targets physically distinct (for similar procedures, see, e.g., Frost, Kugler, Deutsch, \& Forster, 2005; Garcia-Orza et al., 2010; Perea, Abu Mallouh, \& Carreiras, 2010; Perea \& Pérez 2009).

As was indicated above, models that assume that position coding is computed by a general-domain mechanism can accommodate the presence of masked transposi- tion priming across different types of "objects" (Gómez et al., 2008; Logan, 1996). In other words, the pattern of findings for strings of simple, familiar objects (e.g., line drawings) may be, to some degree, similar to that found with strings of letters, digits, or symbols. The idea here is that masked transposition priming will arise because "object" position-encoding is inherently noisy or (for the case of letter strings) from the activation of orthographic word forms (e.g., open bigrams in the SERIOL model). Lack of masked transposition priming will occur if stimuli at the given positions are not processed at all; also, lack of masked transposition priming can arise because position encoding is very precise, which may be due to processing only a single position or to precise encoding across multiple positions. $^{1}$

\section{Experiment 1}

In the present experiment, we examined whether masked transposition effects occur with strings of line drawings of common objects (e.g., $\stackrel{\circ}{*}-\theta$ ). Note that previous experiments on masked transposition priming have employed simple, alphanumeric objects (e.g., letters, digits, symbols, or pseudoletters).

\section{Method}

Participants Forty students from the University of Málaga took part in the experiment in exchange for course credit. All were native speakers of Spanish, had normal or corrected-to-normal vision, and were naive regarding the purpose of the study.

Materials A set of 320 strings composed of four line drawings of common objects each (e.g., $\%$ \& $\square$ ) were used as targets in this experiment. To create the target strings, the following ten line drawings were employed

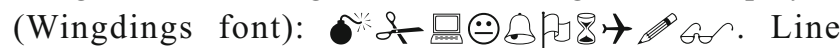
drawings were between $5 \mathrm{~mm}$ (e.g., Go ) and $7 \mathrm{~mm}$ (\&) $10 \mathrm{~mm}$ (o ). Each line drawing was separated by $1 \mathrm{~mm}$ from the preceding and/or the following stimuli in the string. Targets were preceded by primes that were (1) the same except for the transposition of the two initial line drawings (T-initial; e.g., \& $\%$ the same except for the substitution of the two initial line

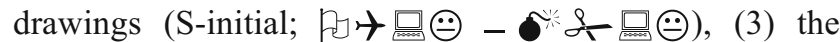
same except for a transposition of the two internal line drawings (T-internal; $\%$ \& $\odot-\%$ of $\square(-)$ ), (4) the

\footnotetext{
${ }^{1}$ We thank Carol Whitney for suggesting this reasoning.
} 
same except for the substitution of the two internal line

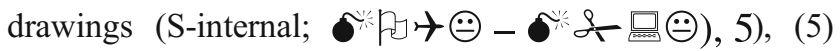
the same except for the transposition of the two final line drawings (T-final; $\%$ \& $\odot \square-$ 学 $\square \odot$ ), (6) the same except for the substitution of the two final line drawings

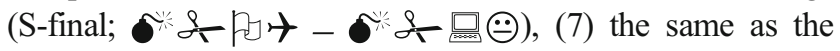
target (identity condition; $\%$ \& $\square \odot-\infty$ \& $\square \odot$ ), and (8) a string of line drawings unrelated to the target (unrelated

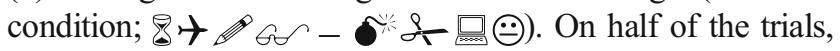
the probe and the target were the same, and on the other half of trials, the probe and the targets were different (e.g., for the

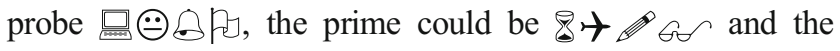
target would be $\$$ ). Eight lists of materials were constructed so that each target appeared once in each list, but each time in a different priming condition. Five participants were presented with each list.

Procedure Participants were tested either individually or in small groups. The stimuli were presented using PCs running the ERTS software for MS-DOS (Beringer, 1999) on a CRT monitor with a 16.6-ms refresh rate. Reaction times (RTs) were measured from target onset until the participant's response. On each trial, a probe was presented above a forward mask consisting of six hash marks (\#\#\#\#\#) for 1,000 ms. Next, the probe disappeared, and the forward mask was replaced by a prime presented for $50 \mathrm{~ms}$, which was replaced by a target. The target stimulus remained on the screen until the response (see Fig. 1).

Participants sat in front of a 15-in. color monitor located at an approximate distance of $60 \mathrm{~cm}$. They were told that they would see strings of line drawings and that they were to press one button if they thought that the probe and target were the same stimulus and to press another button if they thought that the probe and target were different stimuli.
Participants were instructed to make this decision as quickly and as accurately as possible. Participants were not informed of the presence of prime stimuli, and they did not report having seen any prime stimuli when asked after the experiment. Primes and targets were always presented in white on a black background. To avoid visual continuity between the primes and targets (see, e.g., García-Orza et al., 2010), they were presented in a different size-26- and 32point Wingdings font, respectively. The experiment lasted approximately $20 \mathrm{~min}$. Each participant received a different, randomized order of trials.

\section{Results and discussion}

Incorrect responses (2.9\% of the trials) and RTs shorter than $250 \mathrm{~ms}$ or longer than $1,500 \mathrm{~ms}$ (fewer than $0.4 \%$ of the trials) were excluded from the latency analysis. The mean RTs and error percentages in each condition are presented in Table 1. As in previous experiments with the masked priming samedifferent matching task, and given that no masked priming effects are expected in different responses, same and different responses were analyzed separately (see Garcia-Orza et al., 2010; Norris \& Kinoshita, 2008). ANOVAs were conducted on the RTs and error rates, with position (initial, intermediate, and final) and type of prime (transposition vs. substitution) as factors. List was included in the statistical analyses to extract the variance due to the counterbalancing lists (see Pollatsek \& Well, 1995). Planned comparisons of the identity condition versus the transposition conditions and planned comparisons of the unrelated condition versus the substitution conditions were also conducted. For the planned comparisons, $\alpha$ values were corrected using the Bonferroni adjustment (i.e., $\alpha=.05 / 3$ ).

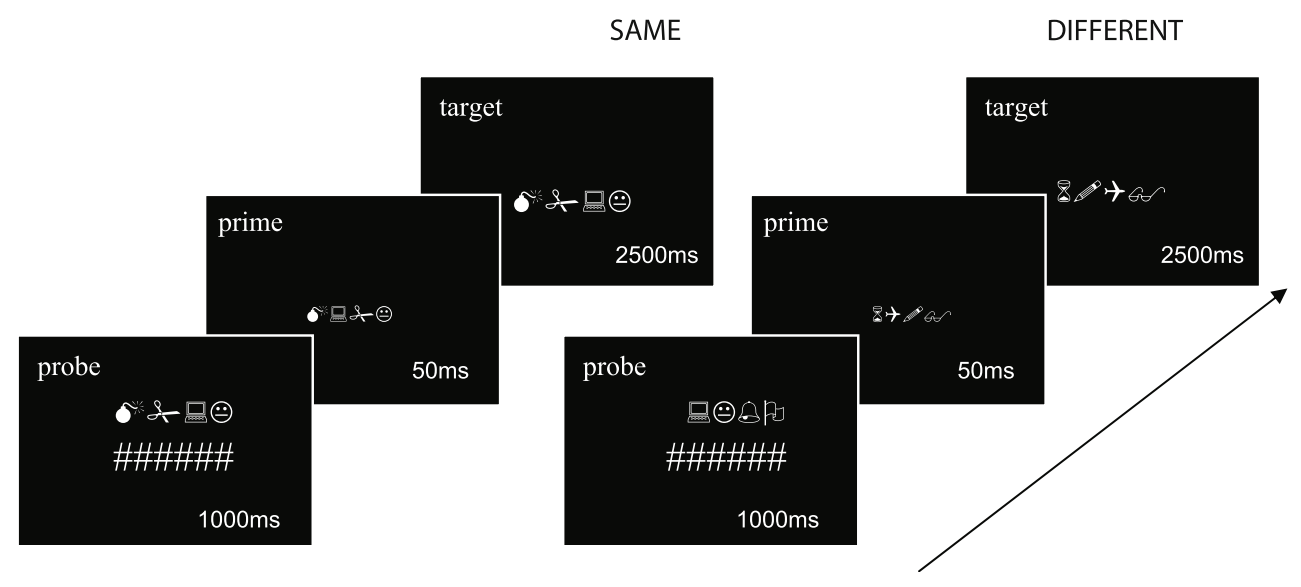

Fig. 1 Description of the visual display used in Experiment 1. A given trial begins with a probe and a mask that are presented simultaneously one above the other for $1 \mathrm{~s}$. Then a prime is presented for $50 \mathrm{~ms}$ (in the same location as the mask), and finally, the target replaces the prime and remains on the screen until the participant's response or until 2,500 $\mathrm{ms}$ has elapsed. In the illustration, an internal transposition prime is presented on both same (left side) and different (right side) trials 
Table 1 Mean response times (in milliseconds), standard errors (in italics), and percentages of errors (in parentheses) for line drawings of common objects in Experiment 1

\begin{tabular}{lllll}
\hline & \multicolumn{2}{l}{ Type of Prime } & & \\
\cline { 2 - 5 } & Transposition & Substitution & Identity & Unrelated \\
\hline Same Responses & & & $55817.1(4.2)$ & $59213.9(4.8)$ \\
Initial position & $58017.7(5.1)$ & $58715.3(5.1)$ & & \\
Middle position & $56816.6(4.8)$ & $56916.1(5.1)$ & & \\
Final position & $57017.2(2.7)$ & $57317.6(6.0)$ & & \\
Different Responses & & & & \\
Initial position & $58614.1(3.2)$ & $58213.9(3.9)$ & & \\
Middle position & $58414.4(4.1)$ & $58814.6(4.5)$ & & \\
Final position & $57813.3(3.5)$ & $58614.5(2.3)$ & & \\
\hline
\end{tabular}

Same responses Neither the main effect of type of prime, $\underline{F}(1,32)=1.04, p=.31$, nor the interaction between position and type of prime, $F<1$, approached significance in the ANOVA on the RTs. The main effect of position was significant, $\underline{F}(2,14)=5.71, p<.01$. Pairwise comparisons showed that targets were responded to $12-15 \mathrm{~ms}$ more slowly when they were preceded by primes modified in the initial position than in internal or final positions (both $\underline{p s}<.03$ ).

In addition, planned comparisons showed that targets preceded by an identity prime were responded to $10-22 \mathrm{~ms}$ faster than were the targets preceded by a transposed-letter prime, although the difference was significant only for the initial transposition ( $22 \mathrm{~ms} ; p<.02)$. In addition, planned comparisons showed that substitution primes behaved like unrelated primes when the replacement was performed in initial position (a 5 -ms difference; $p>.9$ ), whereas substitution primes in internal and final positions produced faster responses than did the unrelated primes (22 and $19 \mathrm{~ms}$, respectively; $p<.01$ and .08 , respectively).

The ANOVA on the error data showed a significant effect of type of prime, $\underline{F}(1,32)=4.46, p<.05$ : Targets preceded by a transposition prime were responded to $1.2 \%$ more accurately than were those targets preceded by a substitution prime. The effect of position was not significant, $F<1$. The interaction between the two factors nearly reached the criterion for significance, $F(2,64)=3.1, p=.05$. This reflected a $3 \%$ difference between transposition and substitution primes in the final position, whereas the parallel differences in initial/middle positions were negligible. None of the planned comparisons between identity primes and transposed primes across each position approached significance (all $p \mathrm{~s}>.3$ ). Likewise, none of the planned comparisons between the unrelated priming condition and the substitution priming condition approached significance (all $\underline{p s}>$.6).

Different responses None of the analyses on the RTs (or percentages of errors) were statistically significant. As was indicated in the introduction, for different trials (e.g.,

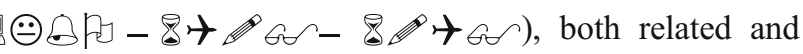
unrelated primes provide evidence that the target is different from the probe, so that no masked priming effects are expected (for similar findings, see Norris \& Kinoshita, 2008; Perea \& Acha, 2009; Perea et al., in press).

The results of this experiment are clear. First, we found no signs of a masked transposition priming effect on the latency data with strings of line drawings as stimuli, thus replicating the data reported by García-Orza et al. (2010) with pseudoletters and by Perea et al. (in press) with Arabic letters for readers with no knowledge of Arabic. Second, we found a small but significant effect of masked transposition priming effect in the error data; this effect was mainly due to the difference in accuracy between targets preceded by transposition primes and targets preceded by substitution primes in the final position (see Table 1; note that this is consistent with the main effect of serial position in the RT data). Third, as usual, there were no priming effects in different responses (see Perea \& Acha, 2009).

The lack of sizable masked transposition priming using common line drawings of objects might be taken to suggest that this phenomenon takes place only with "alphanumeric objects." However, other characteristics of the stimuli, such as their perceptual simplicity, may play a critical role in masked transposition priming. While the models that assume location/perceptual uncertainty across strings of objects (see Gómez et al., 2008; Logan, 1996) remain silent regarding the role of the perceptual complexity of the identity of the constituents, one reasonable assumption is that this factor might play a relevant role in masked transposition priming. The rationale here is that if the identity of an object is not ascertained rapidly, masked transposition priming effects may be negligible, as actually occurred in the present experiment and in the experiment using strings of pseudoletters (see García-Orza et al., 2010; Perea et al., 2011). In Experiment 2, we explored this possibility. In Experiment 2, we examined whether masked transposition priming would occur when strings of geometrical shapes were presented (e.g., +1DD). Geometrical 
shapes are also well-known objects. The critical difference with respect to the stimuli employed in Experiment 1 is that they were perceptually simpler; hence, they allowed us to explore the role of perceptual simplicity in masked transposition priming.

\section{Experiment 2}

\section{Method}

Participants Fifty-six students from the University of Málaga took part in the experiment in exchange for course credit. All were native speakers of Spanish, had normal or corrected-to-normal vision, and were naive regarding the purpose of the study. None of the participants had taken part in Experiment 1.

Materials The manipulation was the same as in Experiment 1, except that, instead of using line drawings of common objects, we employed geometrical shapes. The following ten geometrical shapes were employed to elaborate the strings, using the ITC Zapf Dingbats font:

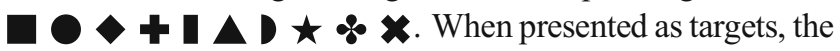
height of all geometrical shapes was $5 \mathrm{~mm}$, and their width varied from $3 \mathrm{~mm}$ for the figures to $6 \mathrm{~mm}$ for the $\Delta \star \star *$ figures. The other shapes were $5 \mathrm{~mm}$ wide. Each geometrical shape was separated by $0.5 \mathrm{~mm}$ from the preceding and/or the following stimuli in the string. Each target - for instance, the target $\mathbf{\Delta} \mathbf{D} \star$ - could be preceded by one of the following primes: $\mathbf{A} \star \star$ (T-initial), $\mathbf{O} \star$

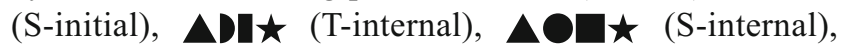
$\Delta \boldsymbol{\Delta} \star \mathbf{D}$ (T-final), $\boldsymbol{\Delta} \mathbf{D O}$ (S-final), $\boldsymbol{\Delta} \boldsymbol{D} \star$ (identity prime), or $4 \mathbf{4}$ (unrelated prime). Eight lists of materials were constructed so that each target appeared once in each list, but each time in a different priming condition. Seven participants were presented with each list.

Procedure The procedure was the same as that in Experiment 1 . To avoid visual continuity between primes and targets (see, e.g., García-Orza et al., 2010), geometrical shapes were presented in a different size-26- and 32-point, ITC Zapf Dingbats font, respectively. The experiment lasted approximately $20 \mathrm{~min}$. Each participant received a different, randomized order of trials.

Results and discussion

Incorrect responses $(6.3 \%$ of the trials) and RTs shorter than $250 \mathrm{~ms}$ or longer than $1,500 \mathrm{~ms}$ (fewer than $0.6 \%$ of the trials) were excluded from the latency analysis. The design and analyses were the same as those in Experiment 1.
Same responses The ANOVA on the RTs failed to show significant effects of type of prime, $F(1,48)=2.39, p=.13$, or position, $F(2,96)=1.27, p=.28$. But the critical finding here is that the interaction between position and type of prime was significant, $F(2,96)=3.68, p<.03$. Simple effect tests revealed that there was a significant masked transposition priming effect in the internal position $(p<.02)$, but not in the initial or in the final positions (both $p$ s $>.6$ ).

Planned comparisons between the identity prime versus the transposition primes (in each position) showed nonsignificant differences of around 2-11 $\mathrm{ms}$ (all $p \mathrm{~s}>.17$ ); that is, transposed primes behaved like identity primes. Finally, planned comparisons between the unrelated prime and the substitution primes (for each position) showed that responses to targets preceded by primes formed by the substitution of the two initial geometrical shapes or the two middle geometrical shapes were around $10-11 \mathrm{~ms}$ faster than the responses to targets preceded by an unrelated prime; the differences were not statistically significant, though (both $p s>.3$ ). Nonetheless, in the final position, targets preceded by a substitution prime were responded to $22 \mathrm{~ms}$ faster than were the targets preceded by unrelated primes $(p<.01)$.

The ANOVA on the error data did not show any significant effects (all $F \mathrm{~s}<1.2$ ). The planned comparisons on the error data also failed to reveal any significant effects (all $p \mathrm{~s}>.39$ ).

Different responses Neither the ANOVAs nor the planned comparisons on the RT/error data revealed any significant effects.

The results of this experiment can be summarized as follows. First, for same responses, we found a significant masked transposition priming effect (i.e., targets were responded to faster when the primes were created by transposing two geometrical shapes than when the primes were created by substituting two geometrical shapes), which was restricted to internal transpositions. Second, planned comparisons between identity primes and transposed primes showed no significant differences (see GarcíaOrza et al., 2010, for similar findings with words, pseudowords, numbers, and symbols). This implies that, at the earliest stages of the processing of the string, the exact localization of geometrical shapes is mainly unavailable (i.e., transposition primes and identity primes behaved in a similar way). Third, we found shorter RTs when targets were preceded by a substitution prime, as compared with an unrelated prime, in the final position; this implies that participants were able to take advantage of the higher perceptual similarity between substitution primes and targets (as compared with unrelated primes) only when the two initial shapes of the prime were identical to the 
corresponding shapes in the target. Fourth, as in Experiment 1 (and in prior research), there were no significant priming effects for different responses. ${ }^{2}$

\section{General discussion}

Previous research has revealed that position coding of alphanumeric objects in a string takes long to encode (e.g., jugde resembles judge; 1492 resembles 1942) and that masked transposition priming occurs for strings of letters, digits, and symbols, but not for strings of pseudoletters (see Garcia-Orza et al., 2010; Perea et al., 2011). To explore the conditions in which masked transposition priming takes place with nonalphanumerical "objects," here we tested whether masked transposition priming occurs with strings of nonalphanumeric objects of different degrees of perceptual complexity: line drawings of common objects (e.g., $\left.\boldsymbol{\sigma}^{*} \rightarrow \boldsymbol{\theta}\right)$ and geometrical shapes (e.g., $\left.\Delta D \star \star\right)$. The main findings can be summed up as follows. First, we failed to find any clear signs of a masked transposition priming effect for line drawings of common objects. That is, a high degree of familiarity with the "objects" that compose the string is not enough to elicit masked transposition priming. Second, masked transposition priming occurred for strings of familiar geometrical shapes (e.g., $\Delta D \star \Delta D \star$ faster than $\Delta O \square \star-\Delta D \star$ ), although the effect was restricted to the internal positions. Thus, the perceptual complexity of the "objects" within a string seems to play a role in masked transposition priming.

The present findings pose some limits to the view that the processes involved in letter position coding are the same as those employed in coding the position of other familiar "objects" (García-Orza et al., 2010). On the one hand, strings of familiar, simple objects (i.e., geometrical shapes) produced a masked transposition priming effect that was restricted to internal positions. Thus, this finding supports the view of an involvement of a general position-coding mechanism that works not only with familiar alphanumeric stimuli (i.e., letters, numbers, or symbols), but also with familiar nonalphanumeric objects - probably, in terms of location uncertainty across strings of objects. On the other hand, the evidence of a masked transposition priming effect

\footnotetext{
${ }^{2}$ As was pointed out by an anonymous reviewer, there is a slight divergence between errors and RTs in the experiment, and this might be suggestive of a speed-accuracy trade-off effect. However, leaving aside the fact that the error data did not show any significant effects in the statistical analyses (i.e., they may just reflect random noise), the critical finding here (i.e., masked transposition priming effect found in internal positions) was not affected by any potential speed-accuracy trade-offs: 602 versus $617 \mathrm{~ms}$ and $9.5 \%$ versus $9.4 \%$, for the transposition and the replacement conditions, respectively.
}

was very weak (i.e., a small effect in the error data) when line drawings of common objects were employed (e.g., * oxperiment 1). This suggests that, using our preferred interpretation, the mechanism responsible for masked transposition priming works only with perceptually simple, familiar objects. For this mechanism to be fast acting and fully operative in a masked priming paradigm, the identity of the objects would need to be ascertained very rapidly. This explains why pseudoletters, which do not have specific "letter/ object" identities, and line drawings, which are perceptually complex, do not show a masked transposition priming effect.

One important question here is whether the pattern of data for strings of simple nonalphabetic objects (i.e., geometrical shapes) differs from that for strings of alphabetic objects. In the present study, masked transposition priming with geometrical shapes occurred only in internal positions. Even though this is a common finding in lexical decision tasks and in normal silent reading (see, e.g., Johnson, Perea, \& Rayner, 2007; Perea \& Lupker, 2003a, 2003b, 2007), the magnitude of masked transposition priming effects for strings of letters, digits, and symbols in the García-Orza et al. (2010) experiments was remarkably similar for initial/middle/final positions with exactly the same task (i.e., a masked priming same-different matching task). Nonetheless, a close look at the data from Experiment 2 reveals that the interaction occurred because the substitution primes in the final position behaved very much like identity primes (see Table 2); otherwise, the data would have revealed a main effect of transposition and no interaction. That is, except for one condition, the data in Experiment 2 were comparable to those reported with strings of alphanumeric symbols by García-Orza et al. (2010).

One implication of the present data is that at the earliest stages of processing a string of objects, there is a general encoding mechanism involved at "object" position coding within a string-regardless of whether the constituent objects are alphanumeric or not. This is consistent with models that assume that there is some degree of location uncertainty in strings of objects (e.g., Ashby et al., 1996; Gómez et al., 2008; Logan, 1996; Ratcliff, 1981). Nonetheless, we cannot discard the existence of a positioncoding mechanism in the word-processing system (i.e., the brain may duplicate some functions). The differences between the pattern of data in the present study (with strings of nonalphanumeric objects) and in the García-Orza et al. (2010) experiments (with strings of alphanumeric objects) does suggest that the underlying processes may not be exactly the same. Indeed, there is some empirical evidence that suggests that there is a brain area that may be sensitive to some type of letter (but not digit) position coding. For instance, Friedmann, Dotan, and Rahamim (2010) reported the existence of individuals with letter position dyslexia (a deficit in which individuals typically make within-word transpositions) who were able to precisely 
Table 2 Mean response times (in milliseconds), standard errors (in italics), and percentages of errors (in parentheses) for geometrical shapes in Experiment 2

\begin{tabular}{lllll}
\hline & \multicolumn{2}{l}{ Type of Prime } & & \\
\cline { 2 - 5 } & Transposition & Substitution & Identity & Unrelated \\
\hline Same Responses & & & $60016.1(7.9)$ & $62815.1(9.7)$ \\
Initial position & $61115.5(9.2)$ & $61815.8(8.0)$ & & \\
Middle position & $60216.0(9.5)$ & $61716.3(9.4)$ & & \\
Final position & $61116.5(7.9)$ & $60616.5(8.6)$ & & \\
Different Responses & & & & \\
Initial position & $60513.4(4.5)$ & $60712.6(4.2)$ & & \\
Middle position & $61012.9(4)$ & $61013.0(3.3)$ & \\
Final position & $60413.8(3.8)$ & $60913.3(3.3)$ & & \\
\hline
\end{tabular}

code digit position within numbers. Friedmann and colleagues employed untimed tasks that did not tap the earliest stages in the process of visual word (or number) recognition, and hence, their task was not well suited to explore the initial position-coding process, which was the focus of the present experiments. Further research should employ a masked priming same-different experiment with strings of letters, digits, and geometrical shapes with patients with letter position dyslexia. One other way to examine the potential similarities/differences in "object" position coding would be to manipulate the location of prime and target stimuli for strings of letters versus strings of other objects (e.g., digits, symbols, geometrical shapes). In this light, Marzouki and Grainger (2008) found that masked repetition priming for centrally located words was greater when primes appeared to the right of fixation than when the primes appeared to the left of fixation. Thus, examining whether or not this pattern of data occurs when strings of objects are used may be another valuable procedure for examining the specificity of the location mechanism in the brain. ${ }^{3}$

It may be of interest to consider an alternative explanation of the data concerning masked transposition priming. ${ }^{4}$ As was indicated in the introduction, lack of masked transposition priming might arise not because the identity of the objects has not been well ascertained (as we have interpreted it), but because position encoding is very precise, which may be due to processing only a single position or to precise encoding across multiple positions. In this view, the pattern of data in Experiment 1 could be explained by assuming that participants generally attended only to the object in the first position. Here is the logic flow: When there was a prime-target mismatch (initial transposition or replacement, unrelated), this increased RTs, relative to a prime-target match (identity prime, transposition/replacement in middle or final positions). However, this reasoning would leave unexplained the fact that the

\footnotetext{
${ }^{3}$ We thank an anonymous reviewer for suggesting this idea.

${ }^{4}$ We thank Carol Whitney for suggesting the following reasoning.
}

targets preceded by an identity prime were responded to 10-22 ms faster than were the targets preceded by a transposed-letter prime. Furthermore, the effects of position are not uncommon in the experiments with the masked priming same-different matching task; for instance, GarcíaOrza et al. (2010) found a disadvantage in initial position with both strings of digits and strings of symbols similar to those in Experiment 1, and masked transposition priming occurred in all (initial, internal, and final) positions for digits and symbols. In Experiment 2, the rationale would be that a broader attentional window could have been at work. Since geometric shapes are visually more similar to each other than the objects are to each other, the strategy of focusing on the object in the first position would be less useful. The consequence would be that participants might have encoded the identities of the internal shapes, rather than their corresponding positions. For same responses, when the prime and target matched both these shapes regardless of their position (internal transposition, identity), RTs were shorter than when the prime mismatched both these shapes (internal replacement, unrelated). On the contrary, when the prime matched one shape and mismatched one shape (transposition/replacement in initial or final positions), RTs in these conditions were similar. However, leaving aside the fact that this reasoning would leave unexplained that the unrelated condition produced the longest RTs (as was expected), it is unclear to us why the identity of the initial position was not encoded in Experiment 2 or why the position of the internal position was not encoded (even though it was allegedly the fixated position). ${ }^{5}$ Furthermore, there is empirical evidence that

\footnotetext{
${ }^{5}$ In order to reconcile the data from Experiment 2 with the existence of masked transposition priming at all positions (initial, middle, final) for strings of symbols (García-Orza et al., 2010), the idea is that punctuation symbols may somehow be associated with orthographic processing (i.e., they are more letterlike than geometric shapes), so that it may be the case that attention is automatically distributed across the entire string, as for strings of letters (i.e., the identities of all the symbols are encoded).
} 
shows that fixation at a given position does not eliminate transposition effects (Gómez, Perea, \& Ratcliff, 2009).

In sum, while the process of letter position coding is a critical aspect of the "front end" of all computational models of visual word recognition, the existence of masked transposition priming for strings of nonalphanumeric objects suggests that some of the mechanisms involved in this process may not be language specific but, rather, may occur at a more general perceptual level. The present experiments have also demonstrated that a factor such as perceptual complexity plays a key role in eliciting masked transposition priming. Further research is necessary to examine the intricacies of position coding in different domains, including the examination of when and where the nature of the linguistic stimuli starts to impose its specificity.

Acknowledgements This research was supported by Grants PSI2008-06107/PSIC and PSI2008-04069/PSIC from the Spanish Ministry of Science and Innovation. We thank Samara Muñoz and Valle Lara for their support in collecting data. We also thank Carol Whitney and two anonymous reviewers for helpful criticism on a previous version of the article.

\section{References}

Ashby, F. G., Prinzmetal, W., Ivry, R., \& Maddox, W. T. (1996). A formal theory of feature binding in object perception. Psychological Review, 103, 165-192.

Baker, C. I., Liu, J., Wald, L. L., Kwong, K. K., Benner, T., \& Kanwisher, N. (2007). Visual word processing and experiential origins of functional selectivity in human extrastriate cortex. Proceedings of the National Academy of Sciences, 104, 90879092.

Beringer, J. (1999). Experimental Run Time System (ERTS) (Version 3.28) [Computer software]. Frankfurt: Berisoft.

Cohen, L., Dehaene, S., Naccache, L., Lehericy, S., DehaeneLambertz, G., Henaff, M. A., et al. (2000). The visual word form area: Spatial and temporal characterization of an initial stage of reading in normal subjects and posterior split-brain patients. Brain, 123, 291-307.

Davis, C. J. (2010). The spatial coding model of visual word identification. Psychological Review, 117, 713-758.

Dehaene, S., Cohen, L., Sigman, M., \& Vinckier, F. (2005). The neural code for written words: A proposal. Trends in Cognitive Sciences, 9 , 335-341.

Farah, M. J. (1994). Specialization within visual object recognition: Clues from prosopagnosia and alexia. In M. J. Farah \& G. Ratcliff (Eds.), The neuropsychology of high-level vision (pp. 133-146). Hillsdale: Erlbaum.

Forster, K. I., \& Davis, C. (1984). Repetition priming and frequency attenuation in lexical access. Journal of Experimental Psychology: Learning, Memory, and Cognition, 10, 680-698.

Friedmann, N., Dotan, D., \& Rahamim, E. (2010). Is the visual analyzer orthographic-specific? Reading words and numbers in letter position dyslexia. Cortex, 46, 982-1004.

Frost, R., Kugler, T., Deutsch, A., \& Forster, K. I. (2005). Orthographic structure versus morphological structure: principles of lexical organization in a given language. Journal of Experi- mental Psychology: Learning, Memory and Cognition, 31, 1293326.

García-Orza, J., \& Perea, M. (2011). Position coding in two-digit Arabic numbers: Evidence from number decision and samedifferent tasks. Experimental Psychology, 58, 85-91.

García-Orza, J., Perea, M., \& Muñoz, S. (2010). Are transposition effects specific to letters? Quarterly Journal of Experimental Psychology, 63, 1603-1618.

Gómez, P., Perea, M., \& Ratcliff, R. (2009, August). Modelling fixation locations in the overlap model of letter position coding. Paper presented at the Joint Convention of the Society for Mathematical Psychology and the European Mathematical Psychology Group, Amsterdam, The Netherlands.

Gómez, P., Ratcliff, R., \& Perea, M. (2008). The overlap model: A model of letter position coding. Psychological Review, 115, 577601.

Grainger, J. (2008). Cracking the orthographic code: An introduction. Language and Cognitive Processes, 23, 1-35.

Guerrera, C., \& Forster, K. I. (2007). Masked form priming with extreme transposition. Language and Cognitive Processes, 23, $117-142$.

Humphreys, G. W., \& Rumiati, R. I. (1998). Stimulus specificity in visual recognition: Agnosia without prosopagnosia or alexia. Cognitive Neuropsychology, 15, 243-278.

Johnson, R. L., Perea, M., \& Rayner, K. (2007). Transposed-letter effects in reading: Evidence from eye movements and parafoveal preview. Journal of Experimental Psychology. Human Perception and Performance, 33, 209-229.

Kinoshita, S., Castles, A., \& Davis, C. (2009). The role of neighbourhood density in transposed-letter priming. Language and Cognitive Processes, 24, 506-526.

Kinoshita, S., \& Norris, D. (2009). Transposed-letter priming of prelexical orthographic representations. Journal of Experimental Psychology. Learning, Memory, and Cognition, 35, 1-18.

Logan, G. D. (1996). The CODE theory of visual attention: An integration of space-based and object-based attention. Psychological Review, 103, 603-649.

Marzouki, Y., \& Grainger, J. (2008). Effects of prime and target eccentricity on masked repetition priming. Psychonomic Bulletin \& Review, 15, 141-148.

Norris, D., \& Kinoshita, S. (2008). Perception as evidence accumulation and Bayesian inference: Insights from masked priming. Journal of Experimental Psychology. General, 137, 434-455.

Norris, D., Kinoshita, S., \& van Casteren, M. (2010). A stimulus sampling theory of letter identity and order. Journal of Memory and Language, 62, 254-271.

Perea, M., Abu Mallouh, R., \& Carreiras, M. (2010). The search of an input coding scheme: Transposed-letter priming in Arabic. Psychonomic Bulletin \& Review, 17, 375-380.

Perea, M., Abu Mallouh, R., García-Orza, J., \& Carreiras, M. (2011). Masked priming effects are modulated by expertise in the script. Quarterly Journal of Experimental Psychology, 64, 902-919.

Perea, M., \& Acha, J. (2009). Does letter position coding depend on consonant/vowel status? Evidence with the masked priming technique. Acta Psychologica, 130, 127-137.

Perea, M., \& Lupker, S. J. (2003a). Does jugde activate COURT? Transposed-letter confusability effects in masked associative priming. Memory \& Cognition, 31, 829-841.

Perea, M., \& Lupker, S. J. (2003b). Transposed-letter confusability effects in masked form priming. In S. Kinoshita \& S. J. Lupker (Eds.), Masked priming: State of the art (pp. 97-120). Hove: Psychology Press.

Perea, M., \& Lupker, S. J. (2004). Can CANISO activate CASINO? Transposed-letter similarity effects with nonadjacent letter positions. Journal of Memory and Language, 51, 231-246. 
Perea, M., \& Lupker, S. J. (2007). La posición de las letras externas en el reconocimiento visual de palabras [The role of external letter positions in visual word recognition]. Psicothema, 19, 559-564.

Perea, M., Moret-Tatay, C., \& Carreiras, M. (in press). Facilitation vs. inhibition in the masked priming same-different matching task. Quarterly Journal of Experimental Psychology. doi:10.1080/ 17470218.2011.582131

Perea, M., \& Pérez, E. (2009). Beyond alphabetic orthographies: The role of form and phonology in transposition effects in Katakana. Language and Cognitive Processes, 24, 67-88.

Pollatsek, A., \& Well, A. (1995). On the use of counterbalanced designs in cognitive research: A suggestion for a better and more powerful analysis. Journal of Experimental Psychology. Learning, Memory, and Cognition, 21, 785-794.

Ratcliff, R. (1981). A theory of order relations in perceptual matching. Psychological Review, 88, 552-572.
Schendan, H. E., Ganis, G., \& Kutas, M. (1998). Neurophysiological evidence for visual perceptual categorization of words and faces within $150 \mathrm{~ms}$. Psychophysiology, 35, 240-251.

Schoonbaert, S., \& Grainger, J. (2004). Letter position coding in printed word perception: Effects of repeated and transposed letters. Language and Cognitive Processes, 19, 333-367.

Tydgat, I., \& Grainger, J. (2009). Serial position effects in the identification of letters, digits and symbols. Journal of Experimental Psychology. Human Perception and Performance, 35, 480-498.

Whitney, C. (2001). How the brain encodes the order of letters in a printed word: The SERIOL model and selective literature review. Psychonomic Bulletin \& Review, 8, 221-243.

Whitney, C. (2008). Supporting the serial in the SERIOL model. Language and Cognitive Processes, 23, 824-865.

Whitney, C., \& Cornelissen, P. (2005). Letter-position encoding and dyslexia. Journal of Research in Reading, 28, 274-301. 